\title{
Algorithm for Calculating the Basic Optimal Parameters of Magnetic Devices of Telecommunication-Information Control Systems
}

\author{
Reshad E. Ismibayli
}

\begin{abstract}
For calculating magnetic devices of telecommunication -information control systems used the optimal directed graph. On the based the directed graph defining optimal parameters of magnetic devices: complex magnetic resistance, optimal value, complex size of magnetizing force of the coil and other.
\end{abstract}

Index Terms-Algorithm, Magnetic Devices, Telecommunication-Information Control Systems, Optimal Parameters, Magnetic Induction, Directed Graph, Optimal Complex Magnetic Resistance, Complex Size of Magnetizing Force of the Coil.

\section{INTRODUCTION}

We consider the synthesis of magnetic devices by defining optimal parameters. The algorithm for calculating the basic optimal parameters of magnetic devices is represented in the following way $[4,5]$ :

\section{METHODS}

1. By the given magnetic induction or another initial condition, proceeding from the operating conditions, and also with regard to constructive considerations find the type of the material, current density $\left(i_{q}\right)$ chosen from the thermal conditions, the number and sizes of air gaps $\left(\delta_{i}\right)$ in the projected magnetic element or device.

2. Construct a directed graph $L(\vec{X}, U)$ reflecting all possible variants of design of the magnetic element (chain) or device, whose vertices correspond to possible values of distribution of magnetic induction $B_{i}$, while the weights of archs $U_{i}$ to possible losses of the area $P_{i}$.

3. In the constructed graph $L(\vec{X}, U)$ determine an optimal directed graph $m(X, U)$ satisfying the minimum value of loss functional and restriction condition.

4. Determine optimal volume of steel of each i-the area of the magnetic core proceeding from the weight of arches $U_{i}$ of the optimal graph $(X, U)$ by the formula:

$$
V_{i}^{*}=\frac{J_{\mu i}^{*}}{A B_{i \text { av. }}^{2}{ }^{K}, \rho_{x i}, f}
$$

Published on December 3, 2019

Author is with Azerbaijan Technical University, Azerbaijan. (e-mail: reshadismibayli@gmail.com) where $J_{\mu i}^{*}$ is the minimum value of the loss functional of the i-th area, corresponding to $U_{i}$ arch of the optimal graph $m(X, U)$.

$i_{\mathrm{av} .}^{*}=\frac{B_{i}^{*}+B_{i+1}^{*}}{2}$ is optimal average value of magnetic induction of the $\mathrm{i}$-th area of the magnetic element or device.

$B_{i}^{*}$ is the optimal value of magnetic induction at the beginning of the i-the area of the magnetic element, corresponding to the vertex $X_{i}$ of the graph $m(X, U)$.

$B_{i+1}^{*}$ is the optimal value of the magnetic induction at the end of the $\mathrm{i}$-th area of the magnetic element, corresponding to the vertex $X_{i+1}$ of the graph $m(X, U)$.

$\rho_{x i}$ is specific reactive magnetic resistance of the $\mathrm{i}$-th area of the magnetic element determined depending on $B_{i \text { av. }}$ and the material of the magnetic core by specific curves.

5. Proceeding from the optimal volume of steel $V_{i}^{*}$ and given the average length of the area $l_{i}$ determine optimal cross-section $S_{i}^{*}$ of the i-the area of the magnetic core:

$$
S_{i}^{*}=\frac{V_{i}^{*}}{l_{i}}
$$

where $S_{i}^{*}=a_{i} \cdot b_{i} \cdot K_{3}$

$a_{i}, b_{i}$ are the width and thickness corresponding to the type of the standard (stamped) plate.

$K_{3}=0,9+0,95$ is steel fill ratio dependent on the kind of isolation and thickness of steel sheet.

6. Determine optimal complex magnetic resistance of steel $Z_{\mu i}^{*}$ of each i-th area of the magnetic core $[2,3]$

$$
Z_{\mu i}^{*}=\left(\rho_{R i}^{*}+j \rho_{x i}^{*}\right) \frac{l_{i}}{s_{i}^{*}}=R_{\mu i}^{*}+j X_{\mu i}^{*}
$$

where $R_{\mu i}^{*}=\rho_{R i}^{*} \frac{l_{i}}{S_{i}^{*}}$ is optimal active magnetic resistance of steel of the $\mathrm{i}$-th area of the magnetic core; $X_{\mu i}^{*}=\rho_{R i}^{*} \frac{l_{i}}{S_{i}^{*}}$ is optimal magnetic resistance of steel.

$\rho_{R i}^{*}, \rho_{x i}^{*}$ are optimal specific active and reactive magnetic resistances of the $\mathrm{i}$-the area of the magnetic core determined according to summary table of calculation formulas or by specific curves depending on the type of the material of the magnetic core and optimal average value $B_{\mathrm{av}}^{*}$.

7. If in the i-the area of the magnetic core there is an air gap, define its magnetic resistance $\left(R_{\mu \delta i}\right)$ 


$$
R_{\mu \delta i}^{*}=\frac{\delta_{i}}{\mu_{0} S_{i}^{*}}
$$

where $\mu_{0}=4 \pi 10^{-7} \Gamma / M$ is magnetic constant of void.

8. If in the i-the area of the magnetic area there is a screen, calculate its complex magnetic resistance

$$
Z_{\mu \ni}=R_{\mu \ni}+j x_{\mu \ni}
$$

where $R_{\mu \ni}$ and $x_{\mu \ni}$ are active and reactive magnetic resistances, respectively, defined by [2].

9. If on the i-the area of the magnetic core there is a magnetizing coil, then by the known formulas define:

a) optimal number of turns $\left(W^{*}\right)$ of the coil with regard to active resistance of the coil $R_{0}$ and steel loss, as

$W^{*}=\frac{U}{\sqrt{C_{1}^{2}+C_{2}^{2}+2 C_{1} C_{2} \sin \theta}}$

where $U$ is the supply voltage;

$C_{1}=\rho l_{\mathrm{av} .} \cdot i_{q}, C_{2}=\frac{\omega}{\sqrt{2}} B_{i}^{*} S_{i}^{*}$ are the coefficients;

$\rho$ is specific resistance of the coil's copper;

$l_{\mathrm{av} .}=2\left(a_{k}+b_{k}\right)+\pi h_{k}$ average length of the turn;

$a_{k}=2\left(a_{1}+\sum \Delta\right), b_{k}=b+2 \sum \Delta$ are frame dimensions;

$\sum \Delta=\Delta_{1}+\Delta_{2}+\Delta_{3}$ are structural sizes of the magnetic element (chain) taking into account the thickness of the coil's frame, air gap between the frame and magnetic core;

$h_{k}$ is the coil winding height;

$\omega=2 \pi f$ is angular frequency;

$\theta=\operatorname{arctg} \frac{\rho_{x i}}{\rho_{R i}}$ is loss angle;

b) the complex size of current in the coil

$I_{k}^{*}=\frac{B_{i}^{*} S_{i}^{*} Z_{\mu i}^{*}}{W^{*}}$

c) complex size of magnetizing force of the coil

$F_{k}^{*}=I_{k}^{*} \cdot W$

d) section of the wire

$q=\frac{I}{i_{q}}$

10. Chose the standard diameter of the wire $(d)$, wire brand, fill factor $\left(f_{m}\right)$ depending on the section of the wire $(q)$.

11. Determine optimal value of the area $Q^{*}$ and length of $l_{k}^{*}$ of the winding window:

$Q^{*}=\frac{W^{*} q}{f_{m}}, \quad l_{k}^{*}=\frac{Q^{*}}{h_{k}}$

12. Determine optimal value of the total complex resistance of the coil:

$$
Z^{*}=\frac{U}{I^{*}},=R^{*}+j X^{*}
$$

where $R^{*}, X^{*}$ are optimal active and reactive resistances (electric), respectively, of the coil.

13. Determine optimal total complex value of the coil power

$$
P^{*}=U I^{*}=P_{a}^{*}+j P_{r}^{*}
$$

where $P_{a}^{*}, P_{r}^{*}$ are active and reactive power of the coil; $I^{*}$ is conjugate current complex.

Thus, the basic optimal magnetic electric and structural parameters of the projected magnetic element or device are determined according to the given algorithm $[4,5]$.

\section{ANALYSIS RESULTS}

As an example assume that we must construct an optimal $Ш$-shaped magnetic element of alternating current with magnetization coil in the average core in the presence of air gaps $\delta_{1}, \delta_{2}, \delta_{3}$ for the given magnetic induction in the average core $B_{0}$, the coil supply voltage $U$, mains frequency $f$. The sketch of the magnetic element was given in the figure.

According to the sketch the magnetic element in divided into 7 areas (Fig. 1) The areas 1,3,6 have air gaps $\delta_{1}, \delta_{2}, \delta_{3}$

The knots of the magnetic elements are denoted and then put on the field of the directed graph in the form of quasiordered set of points from 1 to 6 . Each area is allowed more than two variants of possible distributions of magnetic induction, therefore, on the field of the directed graph along the straight line the points from 1 to 6 are put on (parallel to the points 1-6).

Then the put points are connected with the arches according to the magnetic element topology (Fig. 1) that was represented in the form of a directed graph $L(\vec{X}, U)$ in Fig. 1. that reflects some finite set of variants for designing the Ш_shaped magnetic element. In the constructed graph $L(\vec{X}, U)$ the optimal directed graph $m(X, U)$ corresponding to the optimal variant of the magnetic element is defined according to the algorithm satisfying the optimality criterion and restriction condition.

According to the sketch of the magnetic element (Fig. 1) there are seven calculation areas, therefore the optimization process consists of seven steps. It is assumed that the state of the designed magnetic element corresponding to the vertex $5\left(B_{5}\right)$ of the graph $L(\vec{X}, U)$ (Fig. 1) is known.

In this case, among all the arches $U_{i}$ outgoing from the given vertex $5\left(B_{5}\right)$ and entering the vertex $6\left(B_{6}\right)$ or $6^{\prime}$ $\left(B^{\prime}{ }_{6}\right)$ we determine the $\operatorname{arch} U_{i}$ that has a minimum weight corresponding to the minimum value of losses $P_{i}$ in the area. 


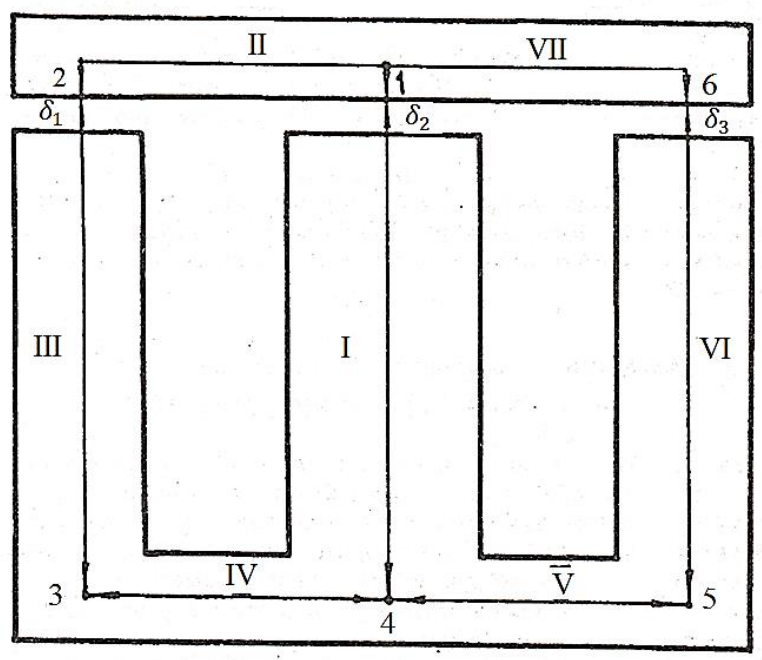

Fig. 1. Sketch of the $\amalg$-shaped magnetic element

It is assumed that the arch $5\left(B_{5}\right)-6^{\prime}\left(B^{\prime}{ }_{6}\right)$ with weight $P_{4}^{V I}$ satisfies the stated condition i.e. $P_{4}^{V I}<P_{1}^{V I}, P_{4}^{V I}<P_{2}^{V I}$.

Thus, minimum losses $P_{4}^{V I}$ and optimal values of induction $B_{5}^{*}$ (at the beginning of the area ) and $B_{6}^{*}$ ( at the end of the area ) correspond to the sixth area.

Then, among all arches entering the vertex $U_{i}$ and outgoing from the vertex $5\left(B_{5}\right) 4\left(B_{4}\right)$-or $4^{\prime}\left(B^{\prime}{ }_{4}\right)$ the arch with minimal weight $P_{i}$ is determined. If assume $P_{3}^{V}<$ $P_{1}^{V}, P_{3}^{V}<P_{2}^{V}, P_{3}^{V}<P_{4}^{V}$ in this case the arch $4^{\prime}\left(B^{\prime}{ }_{4}\right)$ $5\left(B_{5}\right)$ is chosen.

Consequently, minimal losses $P_{3}^{V}$ and optimal values of magnetic inductions (at the beginning of the area) $\mathrm{B}_{4}{ }_{4}-\mathrm{B}^{\prime}{ }_{5}$ correspond to the fifth area of the magnetic element.

Continuing in this way, the optimization process is taken to the vertex $1\left(B^{\prime}{ }_{1}\right)$.

Thus, all the areas of the magnetic element excluding the areas 1 and 7 are optimized. Since in the process of optimization of proceeding areas the vertices $1^{\prime}\left(B^{\prime}{ }_{1}\right), 4^{\prime}\left(B^{\prime}{ }_{4}\right), 6^{\prime}\left(B^{\prime}{ }_{6}\right)$ are determined, then for determining the optimal first area, connecting the vertices $1^{\prime}\left(B^{\prime}{ }_{1}\right)$ and $4^{\prime}\left(B^{\prime}{ }_{4}\right)$, an arch with minimum weight for example the arch $4^{\prime}\left(B^{\prime}{ }_{4}\right)-1^{\prime}\left(B^{\prime}{ }_{1}\right)\left(P_{2}^{I}<P_{3}^{I}\right)$ is chosen, while for determining the seventh area the arch $1^{\prime}\left(B^{\prime}{ }_{1}\right)$ $6^{\prime}\left(B_{6}^{\prime}\right)$ is chosen.

\section{CONCLUSION}

As a result of the done operations the optimal directed graph $m(X, U)$ (Fig. 1) satisfying the chosen optimality criteria and restriction condition is chosen.

Optimal parameters of the given magnetic element are determined by the above algorithm proceeding from optimal distribution of magnetic induction corresponding to the vertices of the directed graph $m(X, U)$ (Fig. 1).

\section{REFERENCES}

[1] Arrays E. A., Dmitri V. M. Modeling of inhomogeneous chains and systems in ECM. M. "Radio i svyaz" 1992.

[2] Barding V.V. Magnetic elements of digital computing machines. M. "Energy" 1984.

[3] Bellman R. Dynamical programming and partial equations. M."M" 1984.

[4] Ismibayli R. E. Optimization of transient processes in magnetic cores of automation of control systems in impulse magnetization. I. AN Az. Rep. ser. fi.-tech. and math. sc. 2000, v. XX, №2-3, p.180-185

[5] Ismibayli R. E. Optimization of transition processes in magnetic cores of automatics equipment of control systems. Proc. of the third inter. sym. math. and comp. appl. 2002, y.V, Konya Turkey pp.35-40 